\title{
Primary lymphosarcoma of the lung with unusual features
}

\author{
ANDREW J. VELIATH, K. K. KHANNA, B. S. SUBHAS, M. R. RAMAKRISHNAN, \\ AND AMRIT L. AURORA
}

From the Departments of Pathology and Paediatrics, Jawaharlal Institute of Postgraduate Medical Education and Research, Pondicherry-605006, India

Veliath, A. J., Khanna, K. K., Subhas, B. S., Ramakrishnan, M. R., and Aurora, A. L. (1977). Thorax, 32, 632-636. Primary lymphosarcoma of the lung with unusual features. Two unusual cases of primary lymphocytic lymphosarcoma of the lung are presented. The first patient is a 5-year-old girl who showed a massive involvement of the entire right lung by tumour. She was treated by radiotherapy followed by cyclophosphamide and was alive and well one year later. The second patient was an adult man who presented clinically with a tumour of the chest wall. Necropsy revealed an extensive direct spread of pulmonary lymphosarcoma with infiltration of the chest wall but without metastatic dissemination or lymph node involvement. Neither primary lymphocytic lymphosarcoma of the lung in a girl of 5 years nor presentation of such a tumour as a chest wall mass in an adult seems to have been described previously.

Primary lymphosarcoma of the lung is an uncommon tumour which characteristically occurs in adults and rarely spreads beyond the pulmonary boundaries (Sternberg et al., 1959; Saltzstein, 1963; Papaioannou and Watson, 1965; Jenkins and Salm, 1971; Rees, 1973). We describe two unusual cases of this rare entity.

\section{Case reports}

CASE 1

A 5-year-old girl was admitted to hospital in March 1975 complaining of cough and breathlessness for two months. On examination she was pale and dyspnoeic. The right chest showed a fullness with a uniformly dull percussion note and markedly diminished breath sounds. The left lung was normal. The trachea and apex beat were shifted to the left. Peripheral lymph nodes were not palpable. All the other systems were normal.

The total and differential leucocyte counts were normal. There were no immature cells in the peripheral blood. Haemoglobin estimation was $11 \mathrm{~g} / \mathrm{dl}$. ESR was $64 \mathrm{~mm}$ fall in the first hour (Wintrobe). Sternal bone marrow aspiration showed a normal picture. Radiological examination of the chest showed a large opacity of the right lung with mediastinal shift suggestive of tumour or massive pleural effusion (Fig. 1). Re- $\stackrel{\mathbb{D}}{\stackrel{D}{\circ}}$ peated attempts at pleural aspiration did not reveal $\overrightarrow{\overrightarrow{0}}$ any fluid in the right pleural cavity. A percutaneous needle aspiration biopsy of the right lung showed a strip of greyish-white tissue diffusely infiltrated by mature lymphocytes without any

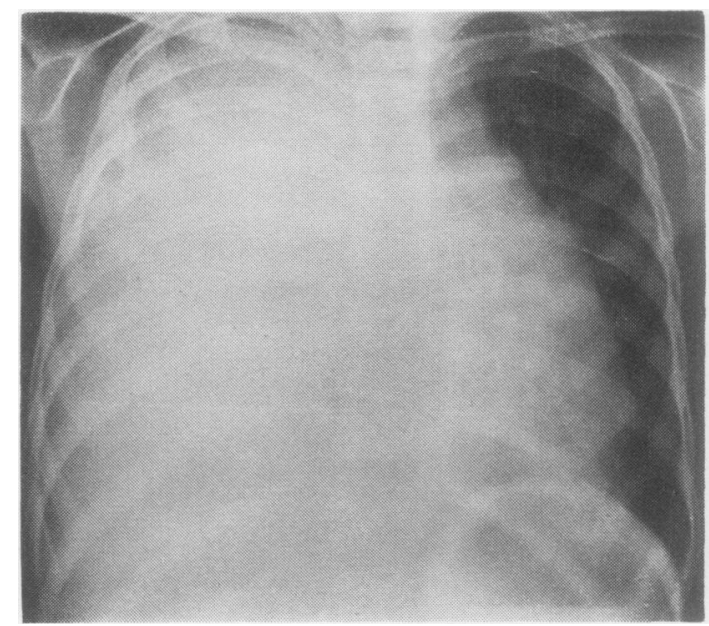

Fig. 1 Case 1. Initial chest radiograph ( $P A$ view) showing a diffuse opacity of the right lung with mediastinal shift. 


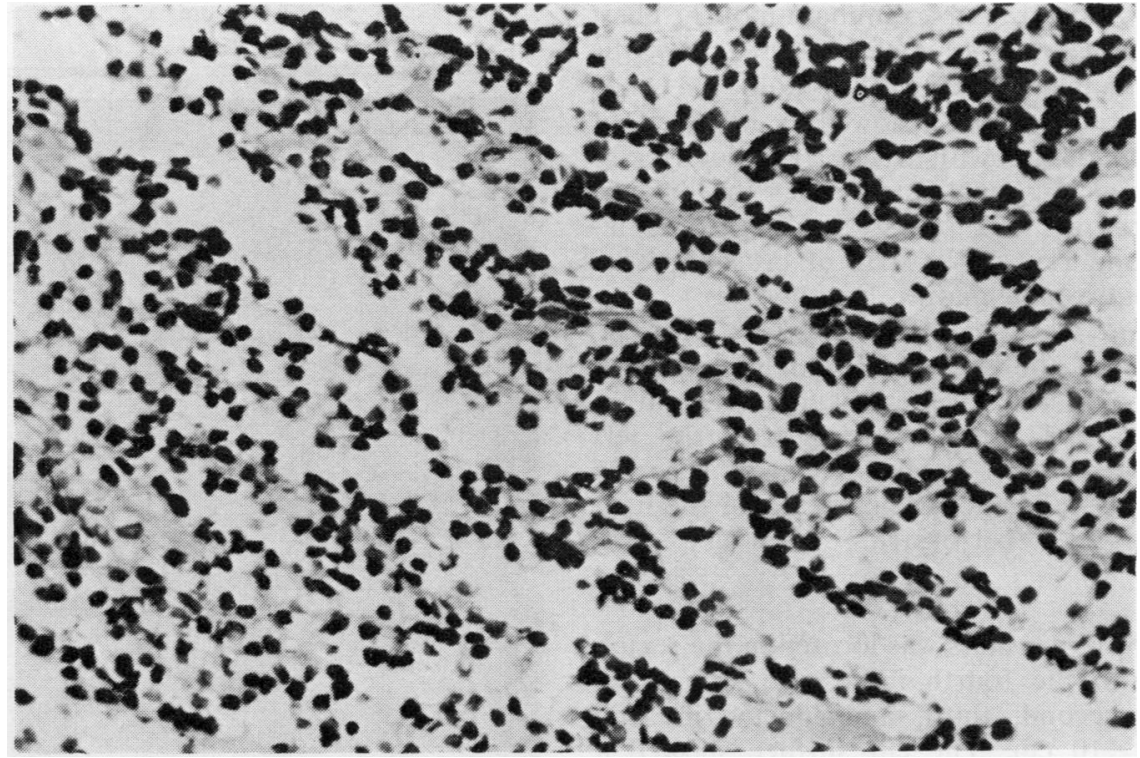

Fig. 2 Case 1. Biopsy of the right lung. There is infiltration by numerous mature lymphocytes without any other cell type (Haematoxylin and eosin $\times 530$ ).

other cell type and was reported as lymphocytic lymphosarcoma (Fig. 2).

The patient was treated with a course of radiotherapy (total $3500 \mathrm{R}$ ) with marked symptomatic relief. A detailed radiological examination with posteroanterior and lateral views following radiotherapy showed a reduction of the right lung opacity and absence of mediastinal shift or lymphadenopathy (Fig. 3). As there was a suggestion of a small effusion in the chest radiograph pleural aspiration was attempted but no fluid was obtained. She was next seen in October 1975 when her general condition had improved. The right lung opacity had markedly reduced in size but was still present. She was treated with a course of cyclophosphamide (Endoxan) therapy. The patient was last seen in March 1976. The lung opacity had reduced further in size and her general condition was good. There was no evidence of spread of the tumour.

\section{CASE 2}

A 35-year-old man was admitted to hospital in March 1975 with complaints of cough, breathlessness, and a painful swelling of the left parasternal region for three months. On examination he was pale, afebrile, and dyspnoeic. The trachea was deviated to the right. The left parasternal mass was well defined, firm, tender, and fixed to the chest wall without involving the skin. The left hemithorax showed a fullness and absence of

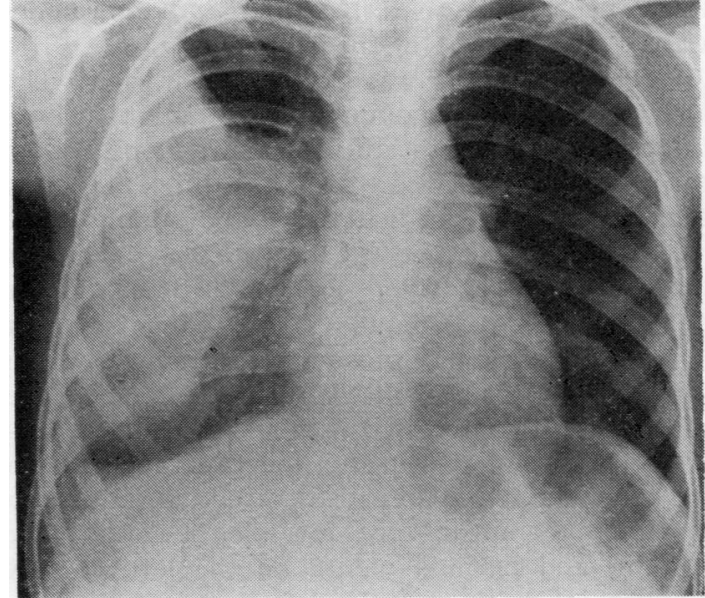

Fig. 3 Case 1. Chest radiograph (PA view) after radiotherapy showing a marked reduction of the right lung opacity without mediastinal shift or lymphadenopathy.

movement with respiration. The percussion note was uniformly dull on the left side. The right lung was normal. Peripheral lymph nodes were not enlarged and all other systems were normal.

The total and differential leucocyte counts were normal. There were no immature cells in the peripheral blood. Haemoglobin estimation was 10.5 $\mathrm{g} / \mathrm{dl}$. ESR was $42 \mathrm{~mm}$ fall in the first hour 
(Wintrobe). Radiographic examination of the chest showed features of massive pleural effusion of the left side with mediastinal shift. Cytological study of the pleural aspirate showed a number of mature lymphocytes with a few red blood cells. Histopathological examination of the needle aspirate from the left parasternal mass was inconclusive and revealed a strip of crushed tissue consisting entirely of mature lymphocytes.

The patient was diagnosed as having a malignant tumour of the chest wall with pleural effusion. On the first day of admission $2000 \mathrm{ml}$ of blood-tinged fluid was aspirated from the left pleural cavity. One week later he became markedly dyspnoeic and cyanosed, dying the next day. Necropsy was performed.

\section{Necropsy}

The left parasternal mass was located in the region of the first to the fourth intercostal spaces, involving the second, third, and fourth ribs and measured $8 \times 10 \mathrm{~cm}$. The cut surface showed a yellowish-white tumour extending from the infiltrated parietal pleura and penetrating the intercostal muscles to form a well-defined mass deep to the pectoral muscles. No enlarged lymph nodes were evident in any of the peripheral sites.

The mediastinum was shifted to the right. The left pleural cavity contained $1000 \mathrm{ml}$ of bloodtinged fluid. The visceral and parietal pleura on the left side appeared yellowish-white, markedly thickened $(1.5 \mathrm{~cm}$ in thickness), and nodular. The left lung was collapsed. The cut surface of the left lung showed a firm yellowish-white tumour measuring $8 \mathrm{~cm}$ in diameter, replacing the upper lobe except for a rim of compressed lung tissue in the lower region (Fig. 4). Bronchi included in the tumour were compressed without mucosal infiltration. The tumour infiltrated the visceral pleura in the upper pole and spread by direct continuity to replace the entire visceral pleura, interlobar septum, and parietal pleura on the left side. A nodular infiltration of the left dome of the diaphragm and pericardium was seen. The left pulmonary artery and vein, left main bronchus, left hilar lymph nodes, oesophagus, and aorta were encircled by tumour without infiltration of their structure. The tumour crossed the midline to the right for a distance of $6 \mathrm{~cm}$ to infiltrate the right parietal pleura and right dome of the diaphragm in their posterior aspect. There was a retroperitoneal extension of the tumour through a bilateral defect between the costal and vertebral origins of the diaphragm with infiltration of both kidneys.

The left lung lower lobe and the right lung were grossly normal. The hilar, mediastinal, and intra-

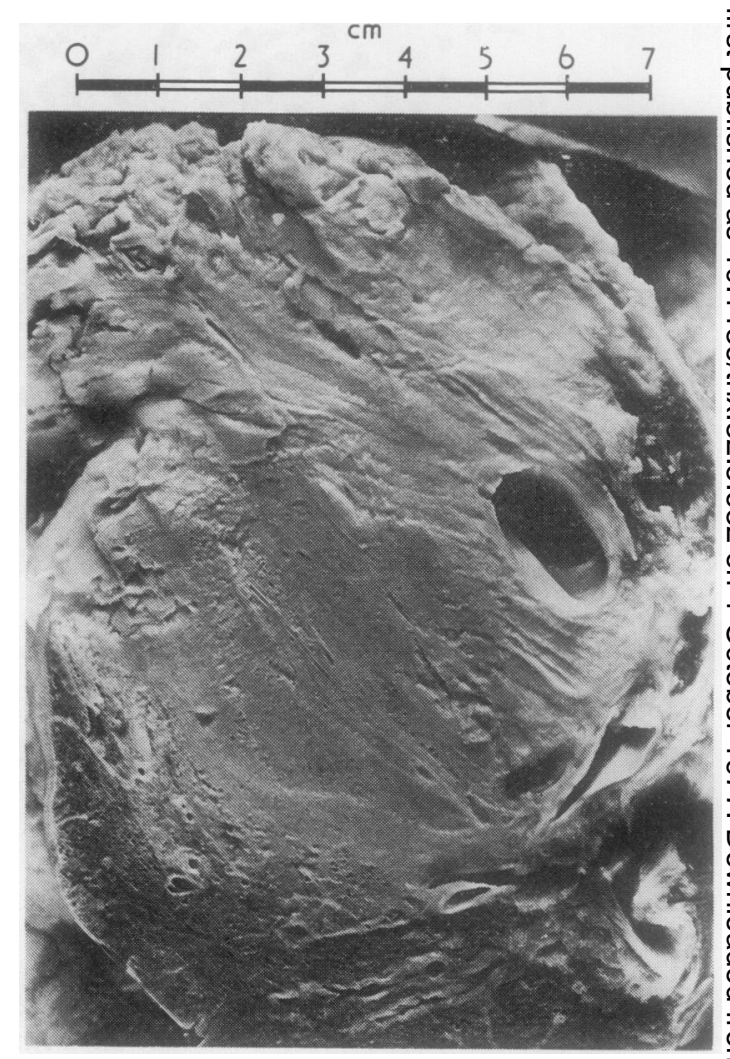

Fig. 4 Case 2. Cut surface of the pulmonary tumour showing a rim of compressed lung tissue in the lower region. A branch of the left pulmonary artery and vein and hilar lymph nodes are encircled by the tumour.

abdominal lymph nodes were of normal size, soft, and free of tumour infiltration. No other organ showed tumour.

Microscopical examination showed the tumour of the upper lobe of the left lung to be unencapsulated and showed a monotonous picture $\frac{D}{0}$ consisting entirely of sheets of mature small lymphocytes (Fig. 5). No other cell type was recog- م) nised. Mitoses were not seen. Germinal centres N were not identified in any of the sections. The NN bronchi showed infiltration of the wall without $\frac{\omega}{\sigma}$ involvement of the basement membrane or mucosa. An identical microscopic picture was seen in sections from the left parasternal mass and $\stackrel{\mathbb{S}}{\rightarrow}$ other sites of gross tumour invasion.

A detailed examination of all the intrathoracic and intra-abdominal lymph nodes showed no tumour infiltration. A study of multiple sections from all other organs, including the right lung, sternal bone marrow, and liver, did not show any tumour. 


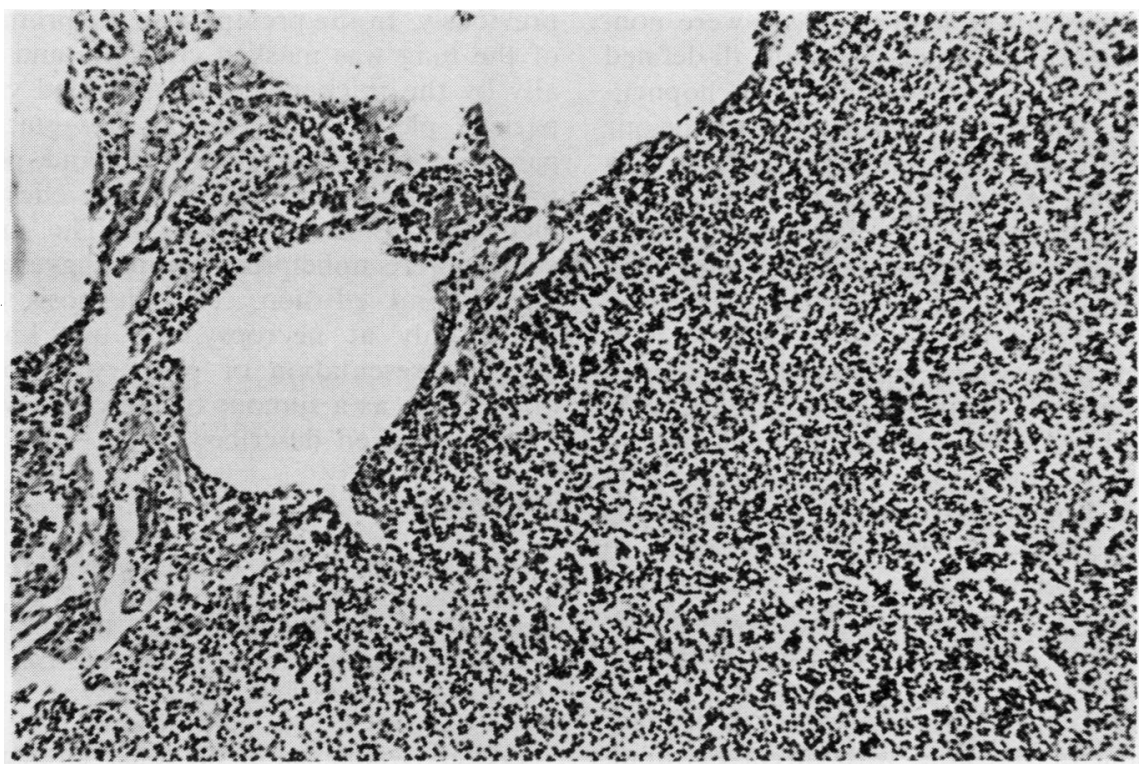

Fig. 5 Case 2. Section from the periphery of the lung tumour showing sheets of mature lymphocytes. Tumour cells are infiltrating the alveolar septa on the left (Haematoxylin and eosin $\times 200$ ).

\section{Discussion}

The definition of primary lymphosarcoma of the lung is still controversial because different criteria have been suggested (Sternberg et al., 1959; Saltzstein, 1963; Papaioannou and Watson, 1965; Jenkins and Salm, 1971; Rees, 1973). It is, however, generally agreed that these tumours usually remain localised to the lungs, except terminally when metastatic dissemination may be observed. Rarely, a direct extrapulmonary spread may occur with slow invasion of contiguous structures (Sternberg et al., 1959). A review of the literature shows that pulmonary lymphosarcoma has been observed mainly in adults of the older age group. Many of the patients are symptom-free, the tumour being detected accidentally on routine chest radiographs (Saltzstein, 1963; Papaioannou and Watson, 1965). In others, respiratory symptoms precede the discovery of the disease by months or years (Papaioannou and Watson, 1965).

Lymphosarcoma occurring in children has been well documented (Dargeon, 1961; Sherman and Wolfson, 1961; Schey et al., 1973). They comprise $10 \%$ of all malignant neoplasms occurring in the paediatric age group and show a marked male preponderance (Dargeon, 1961; Schey et al., 1973). The male to female ratio has varied from $3: 1$ (Dargeon, 1961) to 4.5:1 (Schey et al., 1973). The predominant cell type observed by Schey $e t$ al.
(1973) in a study of 60 children with lymphosarcoma was the lymphoblastic variety $(81.6 \%)$; lymphocytic lymphosarcoma comprised only $10 \%$ of their series. Our first case was of this type. It is of interest that the majority of pulmonary lymphosarcomas occurring in adults are of the lymphocytic cell type (Jenkins and Salm, 1971). Schey et al. (1973) observed that the primary sites of involvement by the lymphocytic variety of lymphosarcoma were the skin, retroperitoneum, groin (lymph nodes), small bowel, parotid gland, and cervical nodes. The lymphoblastic type, in addition, involved the chest, mediastinum, and other extrathoracic sites. The lung as the primary site of involvement by lymphosarcoma was not observed by Schey et al. (1973) and appears from a literature review to be extremely rare in children. Secondary lung involvement, with infiltration of the pleura or lung parenchyma, has been observed (Sherman and Wolfson, 1961; Schey et al., 1973). Pleural spread may occur from a mediastinal lymphosarcoma. Schey et al. (1973) mention that a combination of a mediastinal mass and pleural effusion is strongly suggestive of lymphosarcoma with pleural spread. In the present case the absence of mediastinal lymphadenopathy and pleural effusion ruled against this possibility. Sherman and Wolfson (1961) observed secondary lung involvement in five out of 37 children with lymphosarcoma. The 
radiological appearances in these cases were nonspecific and presented either as small, ill-defined nodular infiltrates or as a patchy bronchopneumonic pattern. The initial chest radiograph of our patient showed a strikingly different picture with a diffuse opacity of the entire right lung. Saltzstein (1963) mentions that radiographic appearances in primary lymphosarcoma of the lung may show a pattern varying from a solitary coin lesion to massive involvement of an entire lung.

To our knowledge this is the first description of primary lymphocytic lymphosarcoma of the lung in a 5-year-old girl. The present case, in addition, shows features uncommon to lymphosarcomas in the paediatric age group. The lymphocytic type of lymphosarcoma is comparatively rare in children as is lymphosarcoma in a 5-year-old girl (Dargeon, 1961; Schey et al., 1973). The combination of both these features in the same patient is extremely unusual.

The tumour in case 2 was diagnosed as primary lymphosarcoma of the lung with direct extrapulmonary spread on the basis of the following features: (1) a gross tumour in the upper lobe of the left lung with compressed but normal bronchi; (2) absence of tumour in the left lung lower lobe and the right lung; (3) spread of tumour by direct continuity without metastatic spread; (4) absence of lymph node involvement; and (5) characteristic microscopic appearance of lymphocytic lymphosarcoma. Generalised lymphosarcomatosis, in contrast, invariably presents with multiple sites of infiltration of the lungs, lesions in other organs, and lymph node involvement.

Direct extrapulmonary spread is uncommon in pulmonary lymphomas. Sternberg et al. (1959) observed such a manner of spread in a reticulum cell sarcoma of the lung with extension to the intrathoracic structures, diaphragm, and liver. The mediastinal nodes were also involved. However, direct spread of a pulmonary lymphosarcoma without lymph node involvement, as observed in case 2, does not appear to have been described previously. In the present case the primary tumour of the lung was masked clinically and radiologically by the thickened and infiltrated visceral and parietal pleura. The clinical presentation with a parasternal mass, on the other hand, pointed to a primary malignant tumour of the chest wall with pleural invasion and effusion. The radiographic studies were unhelpful beyond suggesting a massive pleural effusion. The diagnosis was established only at necropsy. To our knowledge a clinical presentation of primary lymphosarcoma of the lung as a tumour of the chest wall has not previously been described.

\section{References}

Dargeon, H. W. (1961). Lymphosarcoma in childhood. American Journal of Roentgenology, 85, 729-732.

Jenkins, B. A. G., and Salm, R. (1971). Primary lymphosarcoma of the lung. British Journal of Diseases of the Chest, 65, 225-237.

Papaioannou, A. N., and Watson, W. L. (1965). Primary lymphoma of the lung: an appraisal of its natural history and a comparison with other localised lymphomas. Journal of Thoracic and Cardiovascular Surgery, 49, 373-387.

Rees, G. M. (1973). Primary lymphosarcoma of the lung. Thorax, 28, 429-432.

Saltzstein, S. L. (1963). Pulmonary malignant lymphomas and pseudolymphomas: classification, therapy, and prognosis. Cancer, 16, 928-955.

Schey, W. L., White, H., Conway, J. J., and Kidd, J. M. (1973). Lymphosarcoma in children. American Journal of Roentgenology, 117, 59-72.

Sherman, R. S., and Wolfson, S. L. (1961). Roentgen diagnosis of lymphosarcoma and reticulum cell sarcoma in infancy and childhood. American Journal of Roentgenology, 86, 693-701.

Sternberg, W. H., Sidransky, H., and Ochsner, S. (1959). Primary malignant lymphomas of the lung. Cancer, 12, 806-819.

Requests for reprints to: Dr. A. J. Veliath, Pathology Department, Jawaharlal Institute of Postgraduate Medical Education and Research, Pondicherry605006, India. 